ECCOMAS

Proceedia
COMPDYN 2021

$8^{\text {th }}$ ECCOMAS Thematic Conference on Computational Methods in Structural Dynamics and Earthquake Engineering

M. Papadrakakis, M. Fragiadakis (eds.) Streamed from Athens, Greece, 28 - 30 June 2021

\title{
PRELIMINARY NONLINEAR STATIC AND DYNAMIC ANALYSIS OF THE MAIN PYRAMID OF HUACA DE LA LUNA, PERU
}

\author{
Selman Tezcan ${ }^{1}$, Miguel A. Pando ${ }^{2}$, Rafael Aguilar ${ }^{3}$, Benjamin Castañeda ${ }^{4}$, \\ Carol Rojas ${ }^{5}$, Renato Perucchio ${ }^{1}$ \\ ${ }^{1}$ Department of Mechanical Engineering, University of Rochester, Rochester, New York, USA \\ email: selmantezcan@rochester.edu,rlp@me.rochester.edu \\ ${ }^{2}$ Department of Civil, Architectural and Environmental Engineering, Drexel University, Philadelphia, \\ Pennsylvania, USA \\ email: map522@drexel.edu \\ ${ }^{3}$ Department of Civil Engineering, Pontificia Universidad Católica, del Perú - PUCP, Lima, Peru \\ email: raguilar@pucp.edu.pe \\ ${ }^{4}$ Department of Electrical Engineering, Pontificia Universidad Católica, del Perú - PUCP, Lima, Peru \\ email: castaneda.b@pucp.edu.pe \\ ${ }^{5}$ Research Division, Arkeos Asesores y Consultores, Lima, Peru \\ email: carolrojas.vega@gmail.com
}

\begin{abstract}
Huaca de la Luna is a monumental earthen complex located near Trujillo in the North coast of Peru built from $200 \mathrm{AD}$ to $850 \mathrm{AD}$ by the Moche civilization on the slopes of the Cerro Blanco mountain. The principal structure is a massive stepped pyramid built with millions of adobe bricks. The pyramid presents severe structural damage near the $N W$ corner. The static and dynamic response of the pyramid is analyzed using $2 D$ plane strain nonlinear FE models representing the east-west middle section together with the underlying soil profiles. We perform a sensitivity analysis of the static and dynamic response to (a) the pyramid stepped west side profile, (b) the underlying soil and bedrock configuration, and (c) the adobe material tensile strength. Under static conditions the models develop internal fractures but in none of the cases considered these grow to cause the structural collapse of the west side. The west side suffers complete collapse under horizontal accelerations as low as $0.135 \mathrm{~g}$.
\end{abstract}

Keywords: massive earthen construction, archaeological heritage, dynamic analysis, structural failure, non-linear explicit FE analysis, soil-structure interaction. 


\section{INTRODUCTION AND OBJECTIVES}

\subsection{Historical introduction}

The archaeological site of Huaca de la Luna, or Temple of the Moon, is a monumental earthen complex built by the Moche civilization approximately from $200 \mathrm{AD}$ to $850 \mathrm{AD}$ and located near the modern city of Trujillo in the North coast of Peru. Huaca de la Luna was erected on the lower slopes of the Cerro Blanco as part of a sprawling urban center considered to be the capital of the Moche state. The Moche is one of the most important pre-Inca culture, recognized for its distinctive ceramics and architectural decorations [1]. Huaca de la Luna is considered one of the best examples of the Moche cultural achievements because of its well-preserved mural bas-reliefs particularly on the north façade of its main pyramid [2]. After extinction of the Moche culture, circa 850 A.D., the complex was naturally buried under eolian sand deposits, which reduced exposure to seismic loading demands during possible historical earthquakes in that region of Peru. The sand deposits also protected the extensive bas-relief on the north façade from the erosion caused by El Niño torrential rains. The archaeological works that started in the beginning of the 90s has systematically unearthed and exposed the entire Huaca de la Luna complex [2]. Archeologists working at the site interpret Huaca de la Luna as consisting of three pyramids composed by large platforms at various levels, connected by open spaces as reconstructed in Fig. 1(a). Typical of Moche monumental architecture, the complex was entirely built using millions of adobe bricks connected with mud mortar.

The principal structure is a massive stepped pyramid - shown in its present state in Fig. 1(b) - which served for leading political and religious ceremonial events including human sacrifices and elite burials. This pyramid is the result of a succession of construction stages, each consisting in a taller and larger building erected on top of the previous one, so that the new structure encapsulates in its interior all preceding constructions. Each stage was built by erecting adjacent vertical piers of well textured adobe masonry. The pyramid shows signs of extensive anthropogenic and natural damage, primarily in the north and west façade and in the built-up area unearthed by the archaeological excavations on top of the pyramid [2]. A large excavation, attributed to treasure hunting digging during colonial time, cuts deeply in the north façade Fig. 1(b), while several tunnels dug by treasure hunters pierce the west façade at different levels - Fig. 2(b). Structural damage is mainly present in the NW sector of the pyramid including the substantial collapse of the NW corner - Fig. 2(a) - and large cracks in the upper areas which penetrate deeply in the structural system.

\subsection{Research objectives}

The main pyramid is the long term objective of a multidisciplinary study focused on determining the structural behavior of its massive core as well as the built up areas on the top of the pyramid under static and dynamic conditions. Previous work has included a detailed laser-scan survey and 3D reconstruction of the pyramid [3], the mechanical characterization of adobe brick and mud mortar construction through lab testing $[4,5]$ and the geotechnical and geophysical exploration of the foundation soils along the northern façade [6]. These results have been incorporated into a series of preliminary nonlinear FE models of increasing complexity in order to understand how the soil-structure interaction affects the static and dynamic stability of the pyramid. Taking into account the location of the Huaca on the slope of the Cerro Blanco and the results of the geotechnical and geophysical exploration, the FE models assume that the east side of the pyramid is supported on solid bedrock while the west side is built directly on layers of soft sand, with large adobe blocks serving as the foundation layer. Previous research has investigated the relationship between the observed damage and the soft soil support under static 
conditions using a simplified 2D plane strain model derived from the geometry and the ground level of the north façade [7]. In the present research we turn our attention to west side of the pyramid to capture the effect of the original ground level, located $5.5 \mathrm{~m}$ below the north side. To this end, we develop a refined 2D geometry which includes all superimposed construction layers down to the original ground level as revealed by the archaeological excavations along the west side of the pyramid. Adopting a plane strain formulation, this geometry is used to model the structural behavior of an EW cross section positioned in the middle of the pyramid. We then perform a sensitivity analysis of the static and dynamic response to variations in (a) the pyramid stepped west side profile, (b) the underlying soil and bedrock configuration, and (c) the adobe material tensile strength. In each case, the dynamic response is evaluated by applying lateral and vertical ground accelerations leading to the structural collapse of the stepped (west) side of the 2D model. Following a macro-modeling strategy [8,9], the analysis is performed in Abaqus CAE/Explicit using the concrete damaged plasticity formulation to represent a continuum with the mechanical properties of adobe [10]. The soil layers are modelled using the Mohr-Coulomb formulation [11]. The specific material parameters are given below in the modeling section.

a)

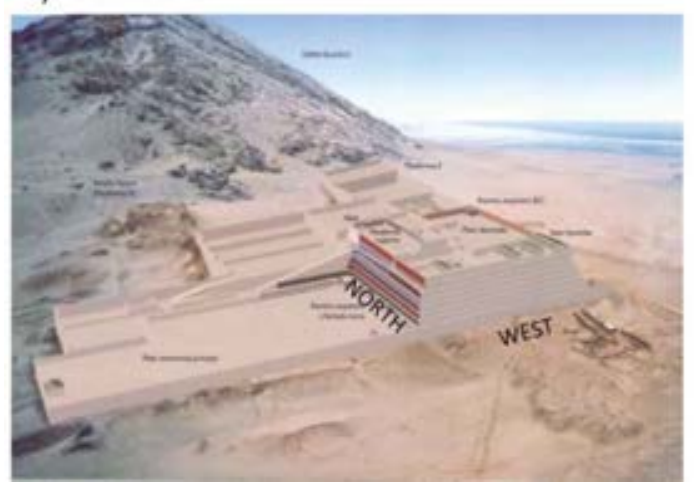

b)

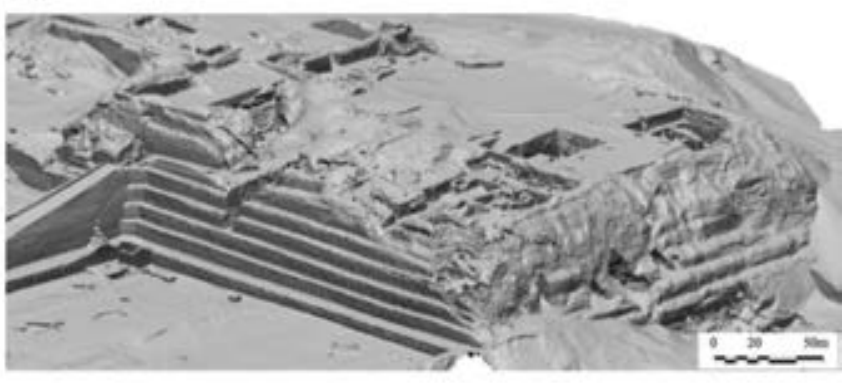

Figure 1: (a) 3D model of Huaca de la Luna [2] and (b) Laser scanning of main pyramid of Huaca de la Luna [3].

a)

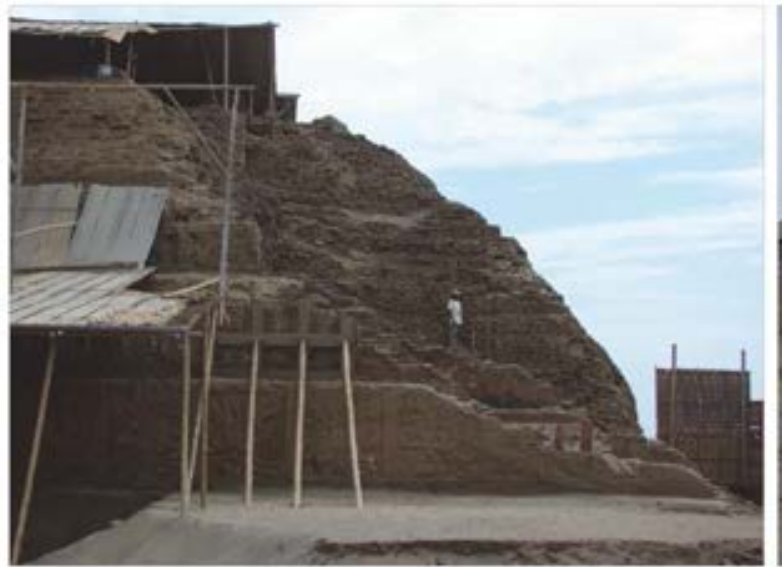

b)

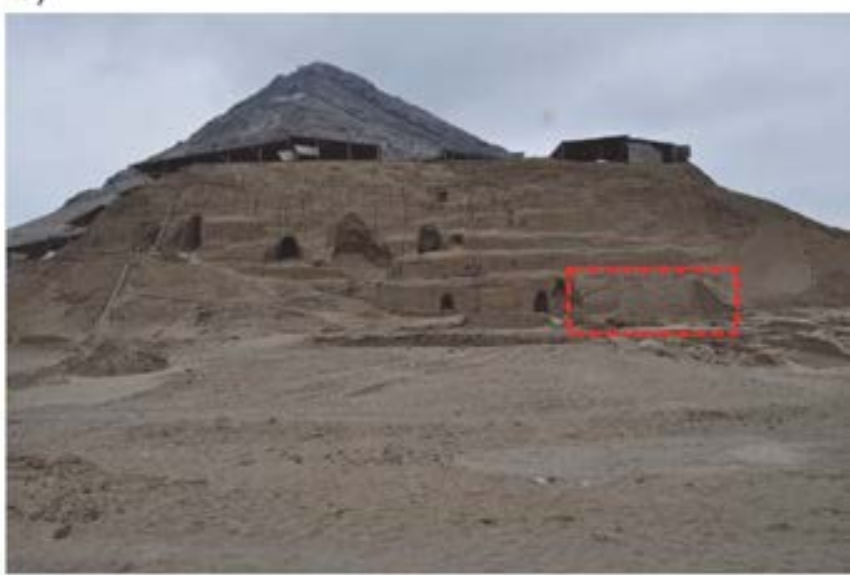

Figure 2: (a) Damaged NW corner of the main pyramid, and (b) West façade of the main pyramid (remaining of

\section{METHODOLOGY} the buttress system is highlighted) [7].

In order to determine the formation of local damage and its evolution into structural failure in the cross-sectional model under static and dynamic conditions, we apply the energy based 
approach previously developed for the failure analysis of other concrete damaged plasticity models $[12,13]$. In brief, the time-evolution of strain (SE), kinetic (KE), and dissipative energy (PD - due to plastic deformations simulating internal fractures) is used to identify the development of local damage conditions up to structural collapse. Consider a simple vertical wall under lateral acceleration normal to the wall - Fig. 3. After gravitational loading, a uniform monotonically increasing horizontal acceleration is applied to the model using the explicit FE formulation available in Abaqus. The desired quasi-static condition is enforced by adopting long time intervals for the application of both gravitation and lateral accelerations: in the example shown, five seconds for gravitation to increase linearly from 0 to $1 \mathrm{~g}$ followed by ten seconds for lateral acceleration to go from 0 to $0.8 \mathrm{~g}$. As long as structural integrity is maintained under increasing lateral acceleration, KE remains negligible during the evolution of quasi-static structural response. Structural failure is associated with the sudden transition from quasi-static to a fully dynamic state and is detected by the accompanying asymptotic growth of KE. PD follows an identical growth pattern, becoming asymptotic at collapse. Notice also that the viscous dissipative energy (VD) produced by the fictitious viscosity parameter used in Abaqus/CAE Explicit to dampen possible oscillatory behavior remains negligible during static conditions and then goes asymptotic at failure.

The examination of the energy plots in Figure 4 suggests complementary ways to associate a specific time interval to failure. KE begins to develop at $7.3 \mathrm{~s}$ and becomes asymptotic at 7.9 $\mathrm{s}$, reaching the $10 \%$ value of the total internal energy (i.e. SE + PD + KE) at $7.7 \mathrm{~s}$. PD, which can be taken to represent the work necessary to propagate the fractures at the base of the wall, begins to appear at $6.6 \mathrm{~s}$ and crosses the $\mathrm{SE}$ at $7.85 \mathrm{~s}$. Thus, purely on energy considerations, total failure can be reasonably defined to happen between $t=7.7 \mathrm{~s}$ (when the loading process cannot anymore be regarded as static) and $t=7.85 \mathrm{~s}$ (when the PD equals the SE).

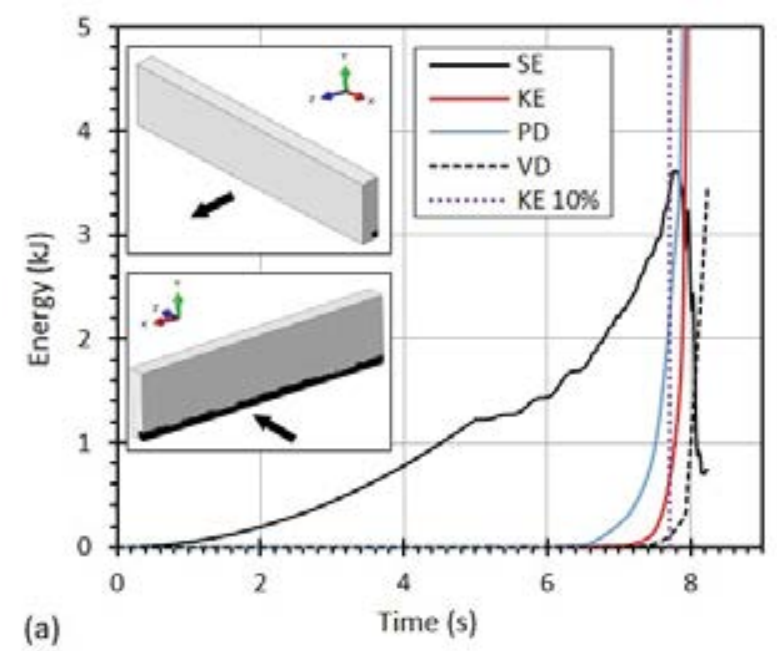

Figure 3: Wall under normal acceleration: energy curves versus analysis time [13].

In the present work, after gravitational loading, lateral accelerations are applied directly to the boundary conditions at bedrock level. In this case, since the entire model is accelerating under inertial forces, KE develops rapidly well before collapse conditions and cannot be used to detect collapse. Under these circumstances, we can still depend on PD to trace the evolution of damage to full collapse, with the latter taken to be the time at which PD equals SE.

The explicit (dynamic) nonlinear FE formulation is preferred over the implicit one because of its capability to sustain large deformations and attendant local material failures, and to capture unequivocally the collapse condition due to lateral accelerations. In fact, in most of the 
cases of the present study, it may be not possible to detect the actual failure condition using an implicit nonlinear FE formulation because the analysis terminates due to numerical error (e.g., the stiffness matrix becomes singular) before the actual structural failure occurs. On the negative side, dynamic explicit models are computationally costly. In order to ensure algorithmic convergences, Abaqus Explicit requires the time increments of the order of $10^{-6}$ second for both gravitation and lateral acceleration.

\section{DESIGN OF MODELS}

The sensitivity analysis of the static and dynamic response of the EW cross section is based on twenty seven models derived from the combination of three variations of the west side stepped profile, three underlying soil and bedrock configuration, and three adobe material tensile strength.

\subsection{West side stepped profile}

Based on the archaeological reconstruction, the original platform level on the west side of the pyramid can be located at approximately $25.2 \mathrm{~m}$ from the ground [14]. Of relevance for interpreting the geotechnical data, we notice that the ground level on the west side is approximately $5.5 \mathrm{~m}$ lower than level of the plaza in front of the north façade. The architecture of the upper west side is well defined in terms of step dimensions. However, due to higher level of damage, less is known with certainty about the original configuration of the lower steps [15, 16]. Thus, working from the archeological cross sections, the upper five steps - extending approximately the $17.1 \mathrm{~m}$ in elevation - can be reasonably reconstructed and matched with the identical steps on the north façade. However, the architectural configuration for the remaining $8.1 \mathrm{~m}$ is ambiguous and can be interpreted as consisting of either three equally spaced steps, or the combination of two irregular steps (a single higher step replacing the two lowest ones from the previous configuration), or even including a triangular buttress at ground level. The resulting three geometrical configurations - hereafter labelled C1, C2, and C3 - are shown in Fig. 4. Notice that the footprint of the stepped west side changes according to the configuration, with $\mathrm{C} 2$ resulting in a substantially smaller footprint than $\mathrm{C} 1$ or $\mathrm{C} 3$.

\subsection{Soil and bedrock configurations}

The reconstruction of the foundation soil configuration below the EW cross section is based on the geotechnical data produced during the exploration conducted in front and to the west of the north façade $[6,7]$. Figure 5 shows the location were cone penetration tests (CPT) were performed and the resulting EW soil stratigraphy configuration with the ground level set at the base of the north façade, i.e., $5.5 \mathrm{~m}$ above the ground level on the west side. Based on additional geological considerations, the stratigraphy illustrated in Fig. 6 is assumed for a NS section taken to the west of the pyramid in correspondence of the CPT02 location (Fig. 5). Starting from these data we construct three alternative bedrock configurations for the EW cross section taken in the middle of the pyramid and referred to the west ground level, see Fig. 6. Configuration B1 assumes the same bedrock position as determined in front of the north façade. For configuration B2, the bedrock layer is shifted uniformly $5 \mathrm{~m}$ down. Configuration B3 is derived from B1 by positioning the bedrock $5 \mathrm{~m}$ lower only in correspondence of second CPT location. As a result, the amount of soft soil below the pyramid in B2 and B3 is larger than B1, while the contact surface between the pyramid and the soft soil is essentially unchanged between B1 and B3 but increases markedly in B2. 

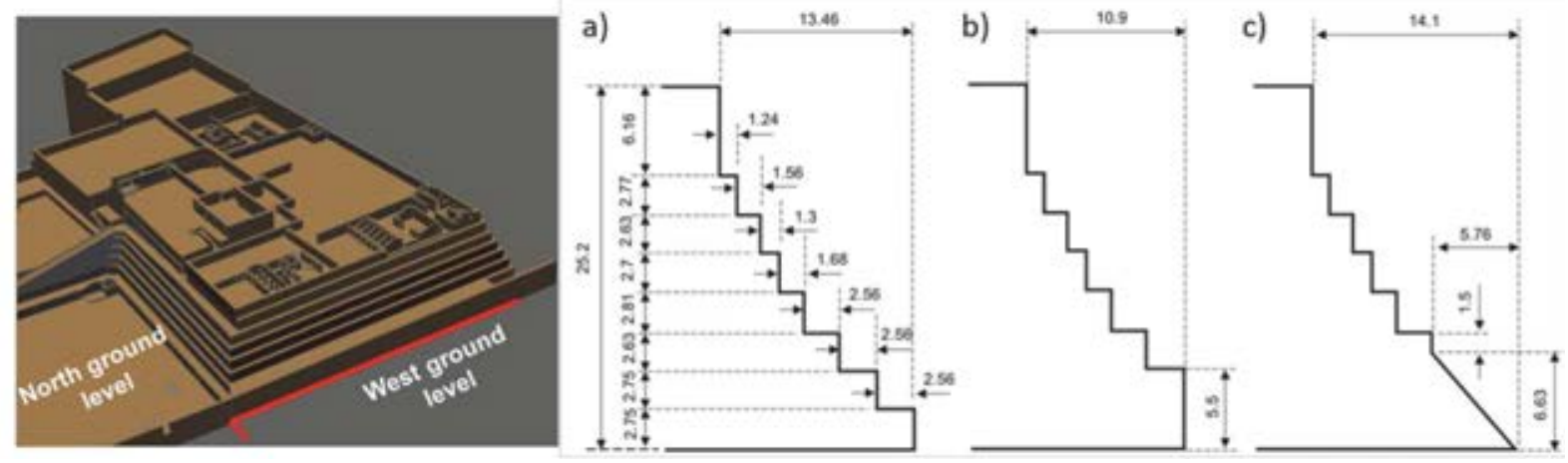

Figure 4: 3D solid model with north and west ground elevation difference on the left, (a) C1 configuration - regular steps, (b) C2 configuration - higher lower step, and (c) C3 configuration - buttress system.
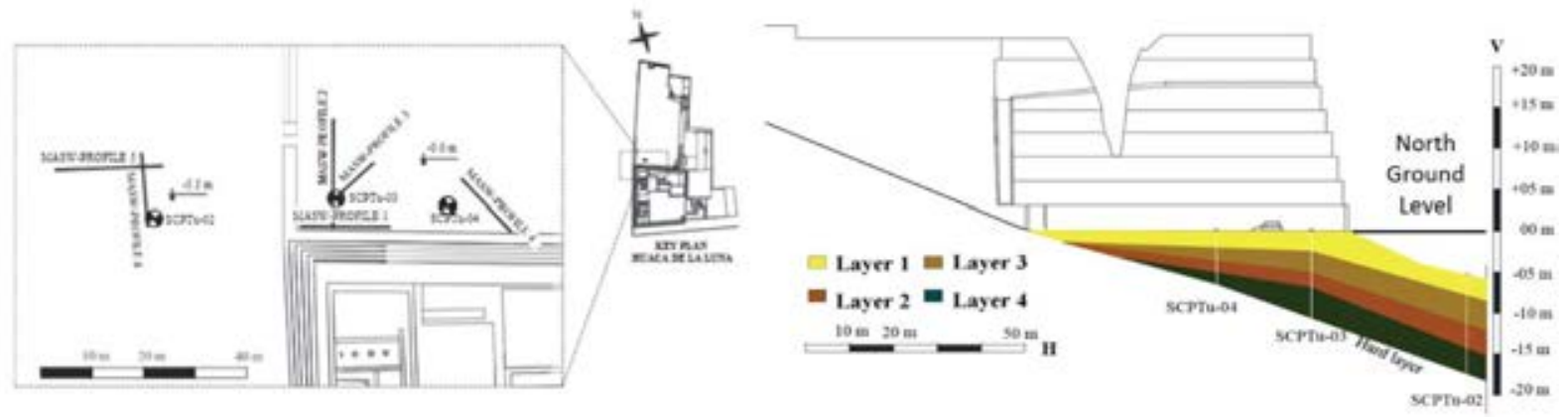

Figure 5: Geotechnical test location (left), bedrock layout and EW soil stratification based on north ground (right) $[6,7]$.
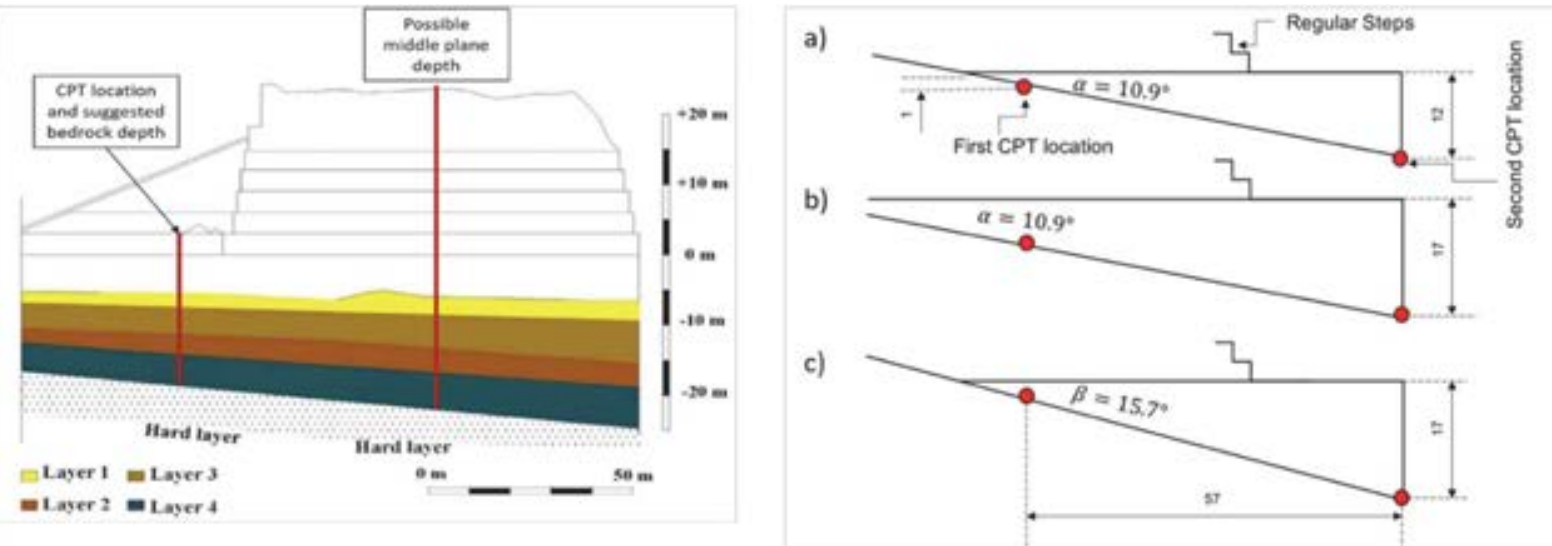

Figure 6: NS bedrock layout and soil stratification (left) [6, 7], and (a) B1 bedrock configuration, (b) B2 bedrock configuration, (c) B3 bedrock configuration.

\subsection{Adobe tensile strength}

In quasi-brittle materials such as adobe masonry, performing tensile tests are extremely difficult. In this case, tensile properties are derived from the compressive strength $\sigma_{c}[8,9]$. For the present case we consider three adobe materials characterized by the same compressive behavior but with tensile strength set as $1 / 10 \sigma_{c}(\mathrm{M} 1), 1 / 20 \sigma_{c}(\mathrm{M} 2)$, and 1/30 $\sigma_{c}(\mathrm{M} 3)$. The postcritical tensile stress versus displacement input for the three cases are shown in Fig.7. 


\section{MATERIAL CHARACTERIZATION}

We use the material parameters for the adobe masonry and the soft soil stratification experimentally given in $[4,5]$ and $[6,7]$, respectively.

\subsection{Concrete damaged plasticity parameters (adobe)}

The concrete damaged plasticity in Abaqus/CAE Explicit requires separate characterization for the elastic, plastic, and inelastic behavior. For the elastic part, the density is $\rho=1735 \mathrm{~kg} / \mathrm{m}^{3}$, the Young's modulus $\mathrm{E}=123 \mathrm{MPa}$, and the Poisson ratio $v=0.2$. The plastic properties are given in Table 1 .

\begin{tabular}{ccccc}
\hline Dilation Angle $\left({ }^{\circ}\right)$ & Eccentricity & $\mathbf{f}_{\mathbf{b}} \mathbf{0} / \mathbf{f}_{\mathbf{c}} \mathbf{0}$ & $\mathbf{K}$ & Viscosity Parameter \\
\hline 1 & 0.1 & 1.16 & 0.67 & $1 \mathrm{E}-8$
\end{tabular}

Table 1: Plastic properties for the adobe material

The inelastic behavior is defined separately for tension and compression. While in [7] the compressive curve is expressed as stress versus total strain, Abaqus requires that the uniaxial compressive response be given in terms of stress versus inelastic strain [10]. The following equation is used to convert the strain data:

$$
\varepsilon_{\mathrm{i}}=\varepsilon_{\mathrm{t}}-\sigma / \mathrm{E}
$$

where $\varepsilon_{\mathrm{i}}$ denotes the inelastic strain, $\varepsilon_{\mathrm{t}}$ the total strain, E the elastic modulus, and $\sigma$ the compressive stress. As in [7] the postcritical tensile response is expressed in terms of stress versus crack-opening displacement. The resulting material curves for inelastic compression and tension are given in Fig. 7. The tensile curves for M2 and M3 are derived by scaling the M1 curve by $1 / 2$ and $1 / 3$, respectively.
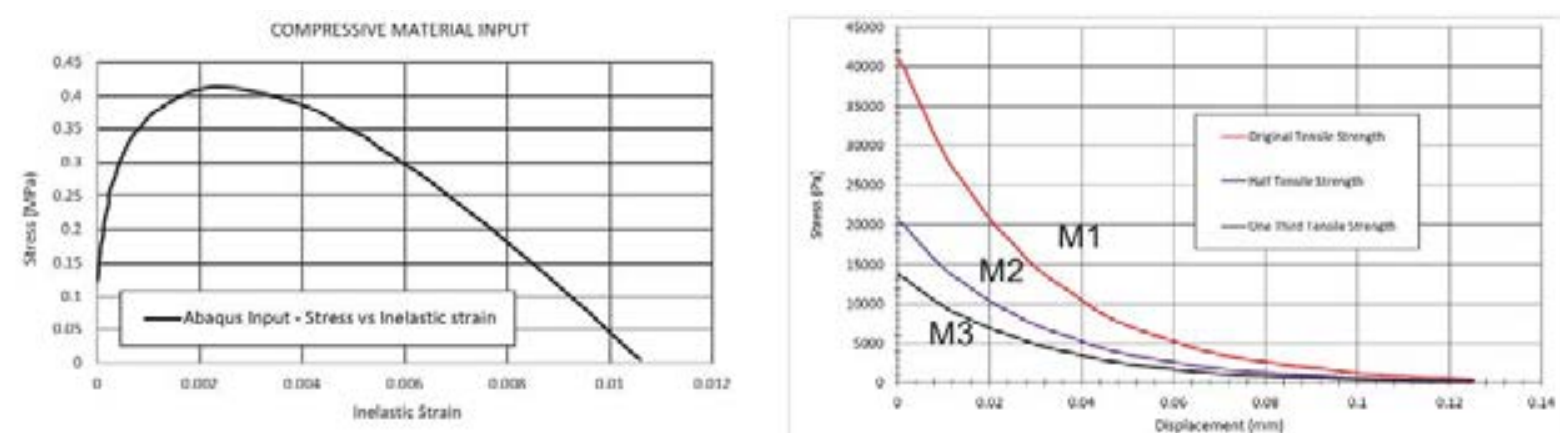

Figure 7: Compressive behavior stress versus inelastic strain (left), material variation on tensile strength as M1, M2 and M3 (right).

\subsection{Mohr-Coulomb parameters (soft soils)}

For the B1, B2 and B3 soil and bedrock configurations defined above, the soil stratification and related properties follow the CPT data given in $[6,7]$. The foundation soils are comprised of layers of eolian sands that range from medium dense near the surface to very dense at deeper depths approaching the bedrock. Each of the four layer shown in Figures 5 and 6 is assumed to behave like a Mohr-Coulomb material with properties given in Table 2. To take into account the marginal cohesion of eolian sand, the cohesion strength of each layer is set to $10 \mathrm{~Pa}$. 


\begin{tabular}{lllll}
\hline Parameter & Layer 1 & Layer 2 & Layer 3 & Layer 4 \\
\hline Mass density $\left(\mathrm{kg} / \mathrm{m}^{3}\right)$ & 1900 & 2000 & 2000 & 2250 \\
Elastic modulus $(\mathrm{MPa})$ & 17 & 43 & 57 & 58 \\
Poisson ratio & 0.43 & 0.43 & 0.42 & 0.41 \\
Friction angle & $40^{\circ}$ & $42^{\circ}$ & $43^{\circ}$ & $43^{\circ}$ \\
\hline
\end{tabular}

Table 2: Material properties of soil layers under Huaca de la Luna [6, 7].

\section{RESULTS}

Although none of the twenty seven M-B-C pyramid configurations reaches structural failing conditions under static loading, all of them exhibit some level of adobe plasticity at the soilstructure interface in proximity to the west edge. The size and intensity of this damage, the additional development of vertically penetrating fractures originating from the top of the pyramid and of damage along the west steps are all affected by the variation in adobe tensile strength and bedrock configuration, and, in a lesser measure, by the step architecture.

We begin by considering how the variation of underlaying bedrock configurations and adobe tensile strength affect material damage under static conditions. Shown in Fig. 8 is the plastic dissipation energy (PD) measured in the adobe mass at the end of gravitational loading. PD increases with bedrock configurations from B1 to B3 and also, as expected, with the adobe tensile strength decreasing from M1 to M3. It is clear that the bedrock configuration has the highest effect on the plastic dissipation energy. Thus, the combined effect of steeper bedrock angle and deeper soft soil foundation - configuration B3 - produces the highest plastic dissipation on the main pyramid. Considering that, as explained earlier, plastic dissipation is directly proportional to the formation of fracture damage in the adobe material, it can be concluded that the bedrock layout makes the most important contribution to material damage under static conditions. This consideration will be further explored by analyzing the results of the various models built with combination of bedrock layout, step architecture, and material tensile strength.

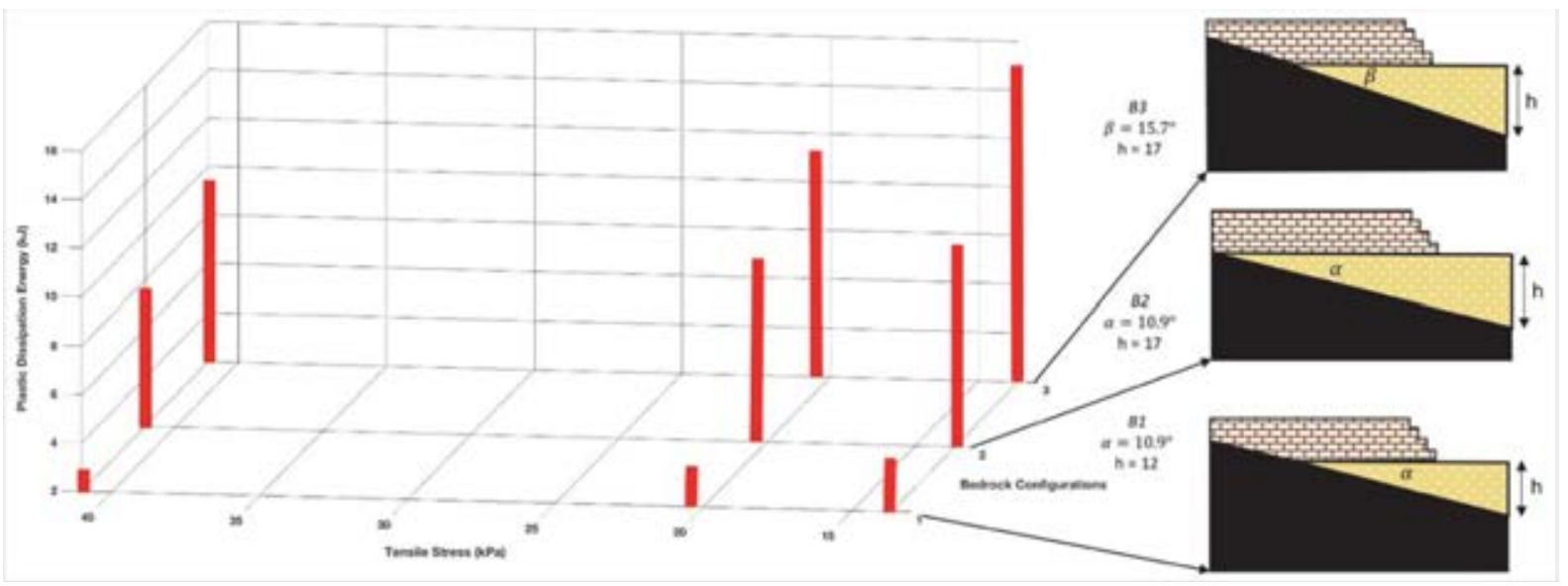

Figure 8: Plastic dissipation energy for material and bedrock variations.

The distribution of maximum principal plastic strains on bedrock configurations B1, B2, and B3 with the highest tensile strength of adobe (M1) are shown in Fig. 9 (a)-(c). These are compared with the results for the same configurations but with the lowest tensile strength (M3), Fig. 9 (d-f). The black zones indicate areas where the strains are above the $10^{-3}$ threshold, and thus where fracture damage can be predicted. For all the M1 cases, compressive stresses - although remaining below compressive strength - produce a nucleus of low level tensile plastic strains at the base of the pyramid in proximity of the west edge-see B3, Fig. 9(c). For B1 and B2 their 
magnitude is below $2.5 \mathrm{e}^{-4}$ and thus not visible in Fig. 9(a) and (b). While there is no fracture development on the top level of the pyramid for B1, a downward vertical fracture forms at the angle between the top platforms for B2 and B3.
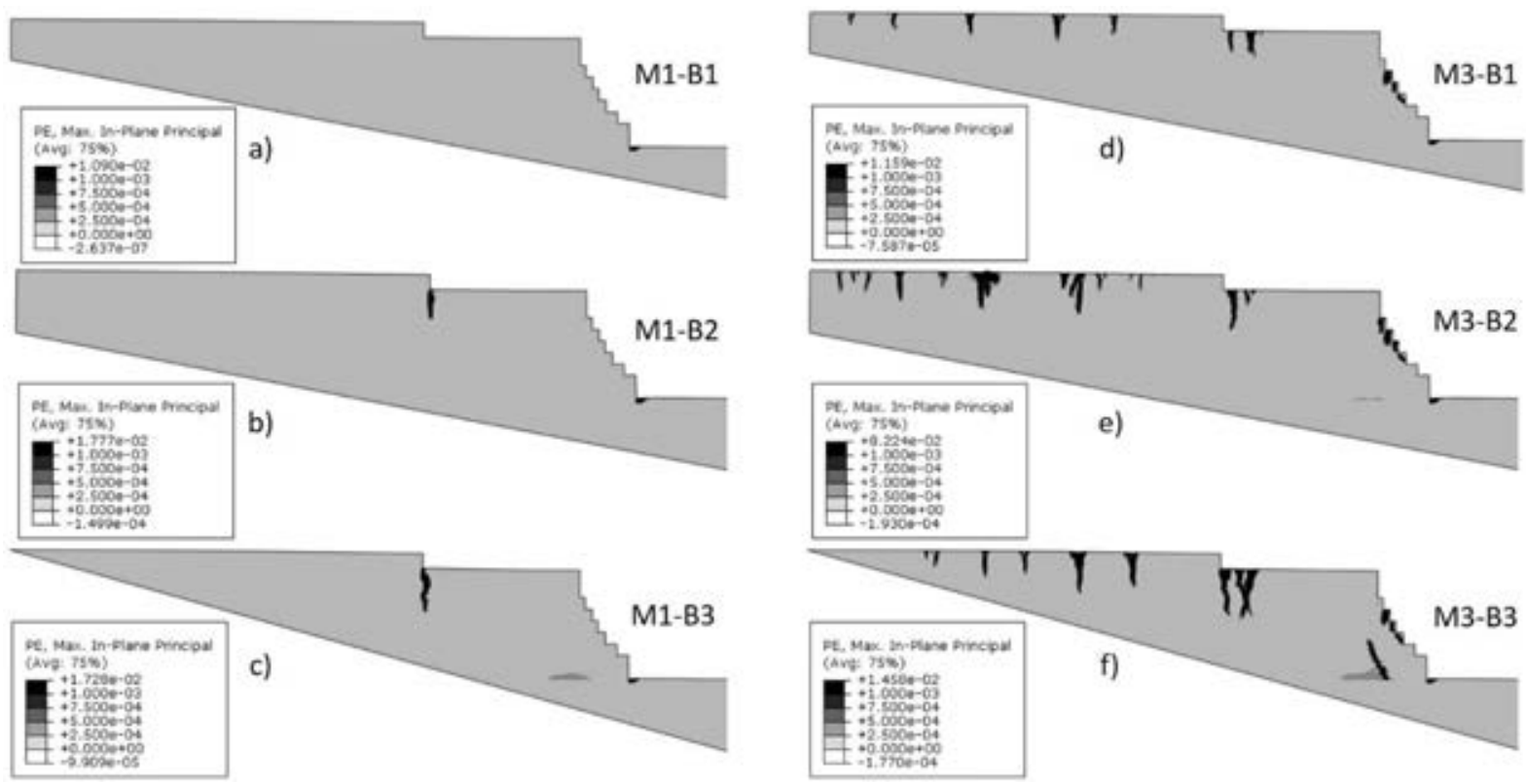

Figure 9: Plastic strain results of bedrock variation for M1 (a-c) and M3 (d-f).

As shown in Fig. 9(d)-(f), the M3 models under static conditions exhibit similar damage patterns at the base of the pyramid. In all three bedrock configurations, however, the downward fractures on the top level increase noticeably in number and in depth and damage appears along the steps on the west side of the pyramid. For M3-B3, a substantial fracture propagates upward from the base near the west corner - Fig. 9(f).

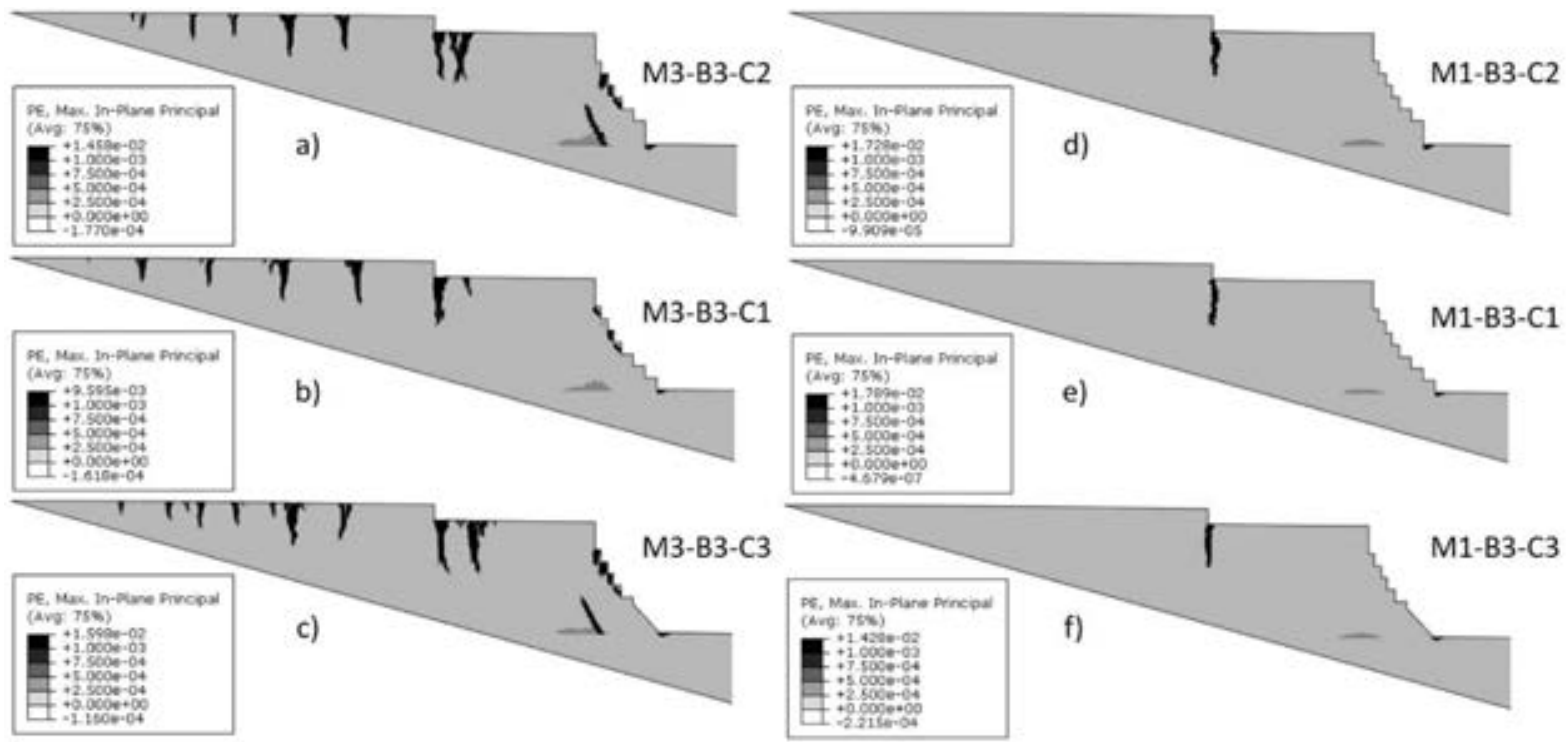

Figure 10: Plastic strain results of step architecture variation for M1-B3 (a-c) and M3-B3 (d-f).

Figure 10 shows the effect of the step architecture on the B3 configurations with material $\mathrm{M} 1$ and M3 under static conditions. The regular step architecture $(\mathrm{C} 1)$ produces the least damaged conditions. For the M3 cases, $\mathrm{C} 1$ appears to stabilize the west façade by containing the 
expansion of the fracture zone at the base, while for the M1 cases, it produces the least plastic dissipation energy. The downward fractures at the top platforms are consistent with those shown in Fig. 9 for M1 and M3. For the M1 cases, there is a single fracture at top of the pyramid, which is not affected by the step architecture. All M3 cases show several downward penetrating fractures at the same location. In addition, the M3-B3-C2 and M3-B3-C3 cases show more extensive damage along and in the proximity of the west steps.
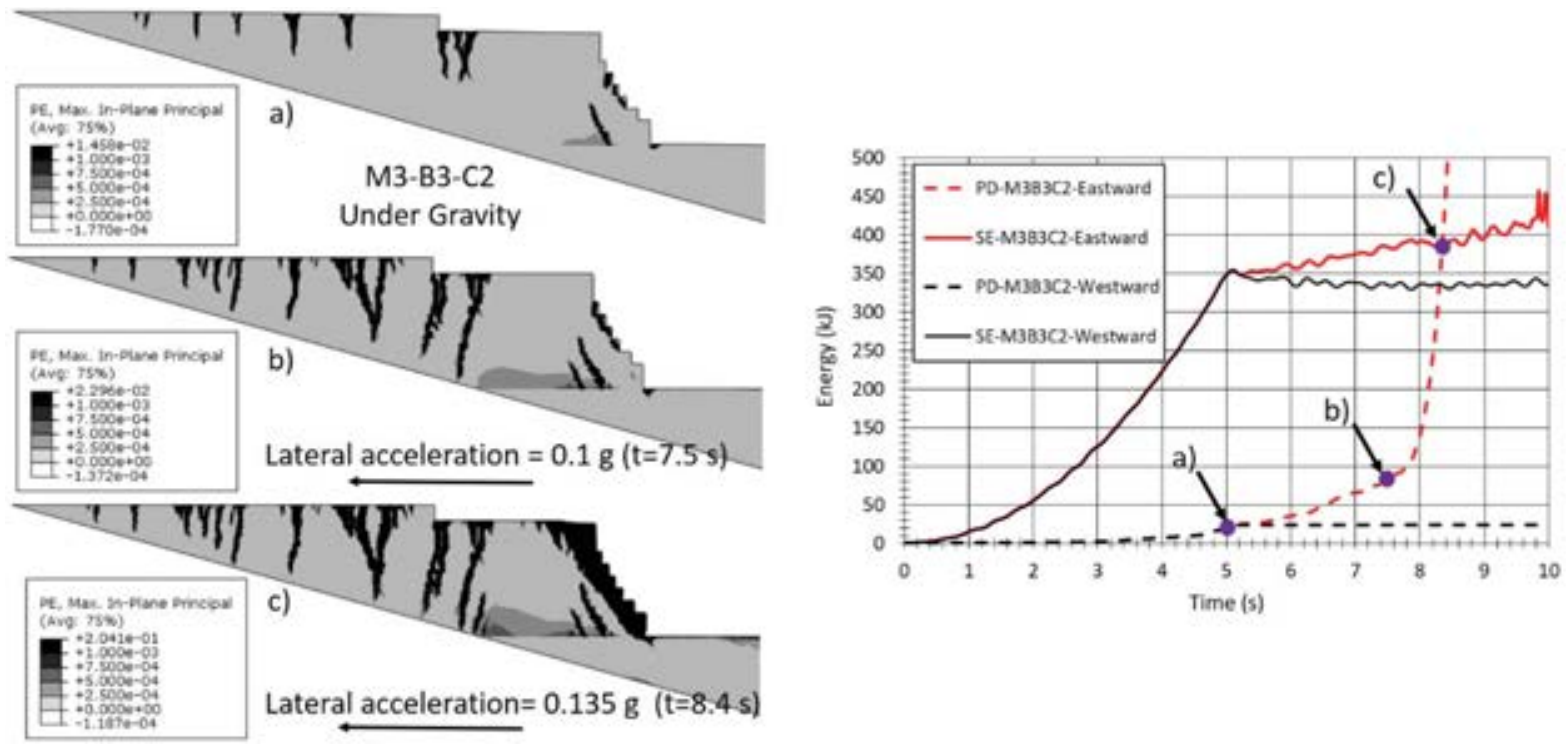

Figure 11: Plastic strain results of M3-B3-C2 configuration under gravity and eastward acceleration (a-c) on the left, energy results of both eastward and westward acceleration cases on the right.

We now examine the effect of horizontal acceleration on the M3-B3-C2 configuration, which exhibits the highest level of structural damage under gravitational loading. The horizontal acceleration is applied at the bedrock level after gravitational loading as a linear function of time, gradually increasing from $0.0 \mathrm{~g}$ to $0.2 \mathrm{~g}$ over 5 seconds. In order to account for the asymmetry of the model, two separate tests are performed with the acceleration applied eastward in one case and then westward in the other. As expected, the results indicate that the pyramid is critically vulnerable under the eastward acceleration as clearly shown by the difference in PD energy curves for the two cases - Fig. 11. The effect of the eastward acceleration is shown in Fig. 11 in terms of damage progression starting at $5 \mathrm{~s}$ (at the end of gravitational loading, Fig 11(a)), at the beginning of collapse at $7.5 \mathrm{~s}$ (horizontal acceleration $0.1 \mathrm{~g}$, Fig. 11(b)), and at full collapse at $8.4 \mathrm{~s}$ (horizontal acceleration $0.135 \mathrm{~g}$, Fig. 11(c)). The structural collapse involves the separation of the entire west side of the pyramid. In contrast, the energy curves for the westward case reveal that the structural situation remains essentially unchanged from the static condition through the following $5 \mathrm{~s}$ of increasing horizontal acceleration.

To explore the effect of horizontal and vertical accelerations applied simultaneously to the M3-B3-C2 model after gravitational loading, a downward acceleration is also added as a ramp increasing from $0.0 \mathrm{~g}$ to $0.1 \mathrm{~g}$ over the same $5 \mathrm{~s}$. The vertical acceleration is selected as half of the horizontal one in consideration that earthquake vertical peak ground accelerations in this aera are generally significantly lower than the horizontal component. As shown in Figure 12, the results demonstrate that the addition of downward acceleration changes the structural failure condition only marginally, with the horizontal acceleration at failure shifting from $0.135 \mathrm{~g}$ to $0.139 \mathrm{~g}$. 

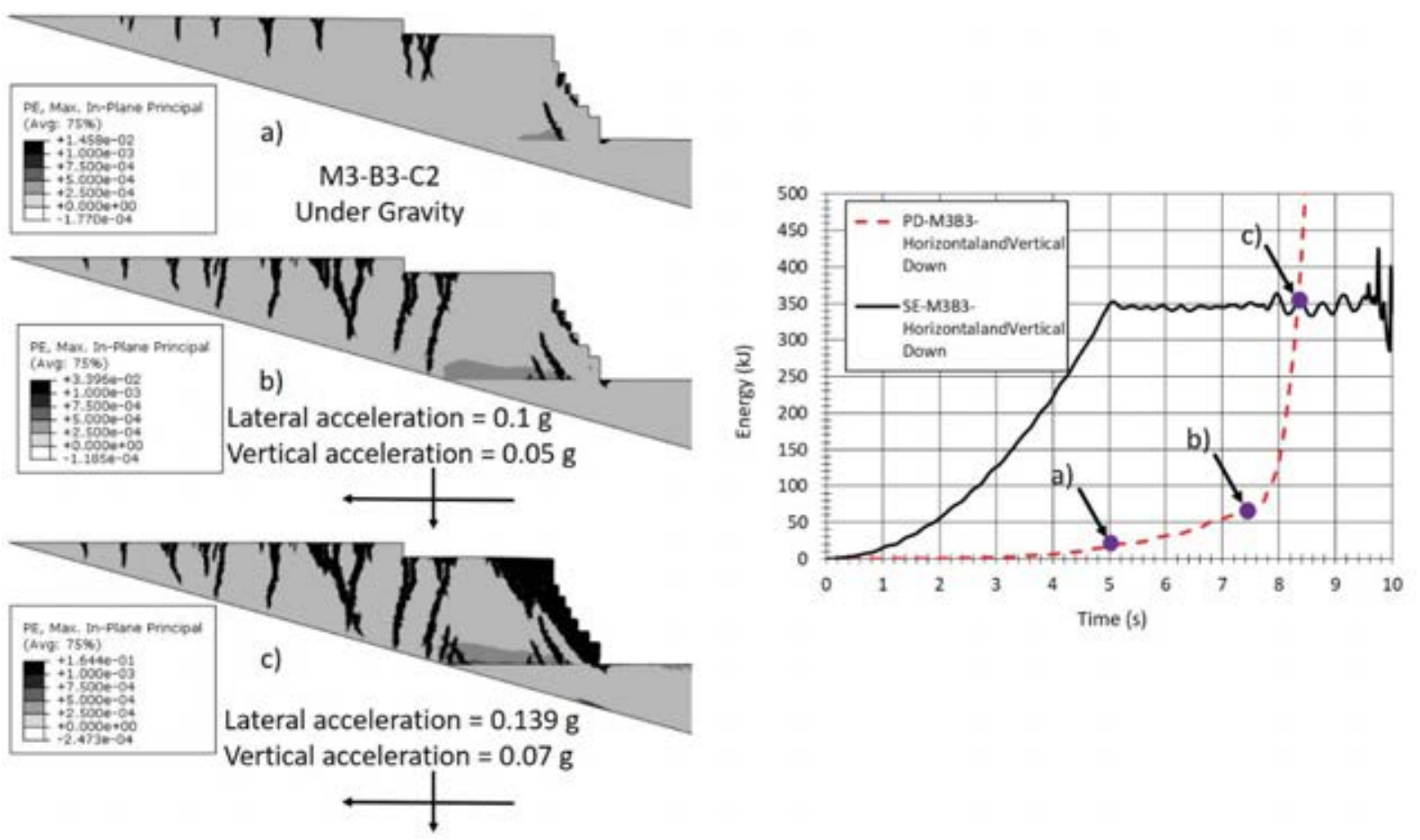

Figure 12: Plastic strain results of M3-B3-C2 configuration under gravity and eastward and downward acceleration (a-c) on the left, energy results on the right.

Figure 13 illustrates the effect of maximum adobe tensile strength as in the M1-B3-C1 case under eastward horizontal accelerations. The lateral capacity of the structure is further increased from $0.135 \mathrm{~g}$ to $0.24 \mathrm{~g}(78 \%$ more than M3-B3-C2). Finally, Fig. 14 illustrates the effect of the step configuration on the M3-B3 model under horizontal accelerations. The comparison with Fig. 11 is revealing. The presence at the ground level of a buttress (C3) or an addition step (C1) increases the lateral capacity by $33 \%$ and $45 \%$, respectively.
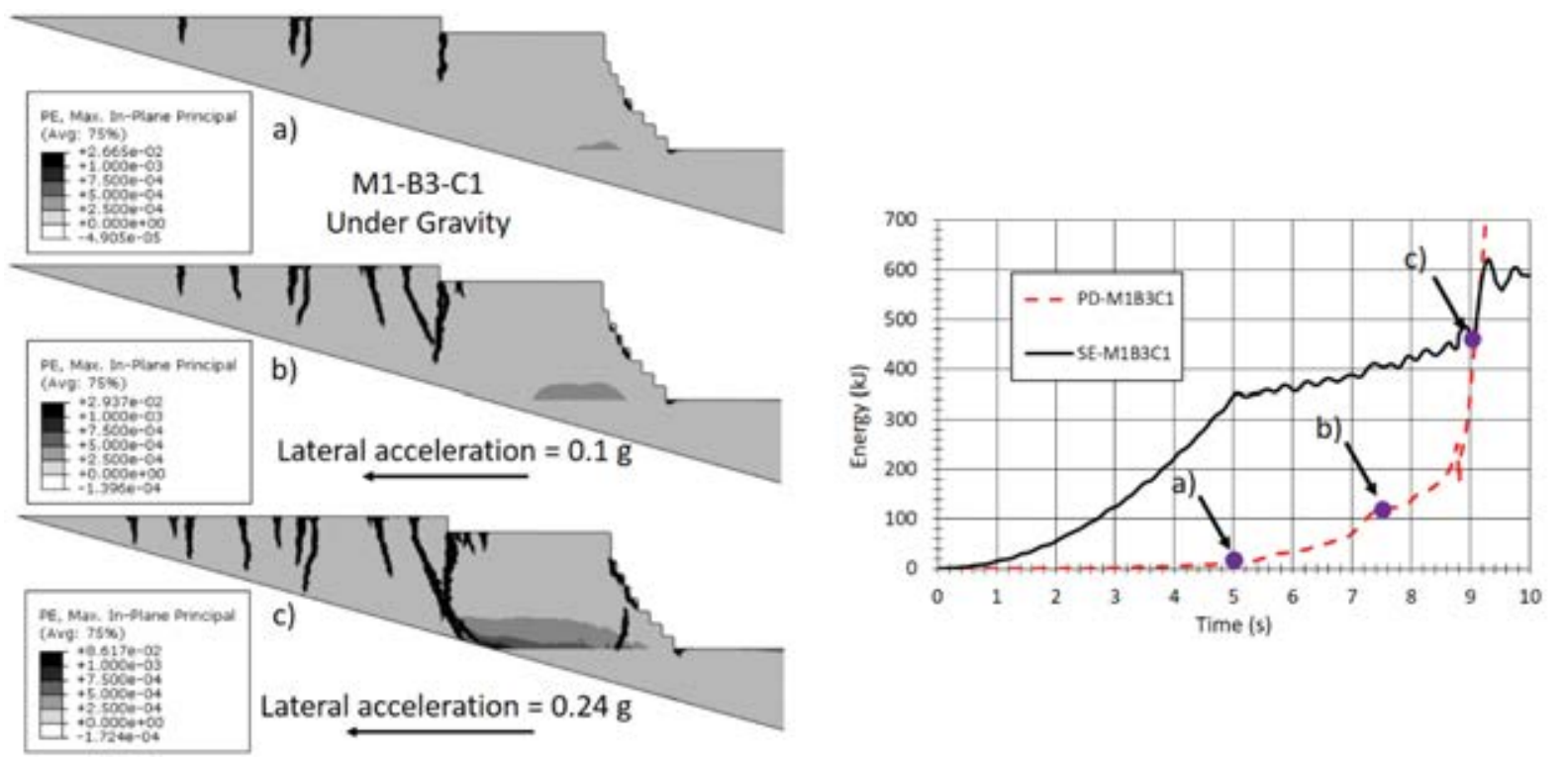

Figure 13: Plastic strain results for M1-B3-C1 configuration under gravity and eastward acceleration (a-c) on the left, energy results on the right. 
It is worth noting the similarity in the failure pattern along the west side between all the M3B3 model and the actual collapse present on the NW corner of the pyramid, Fig. 14.

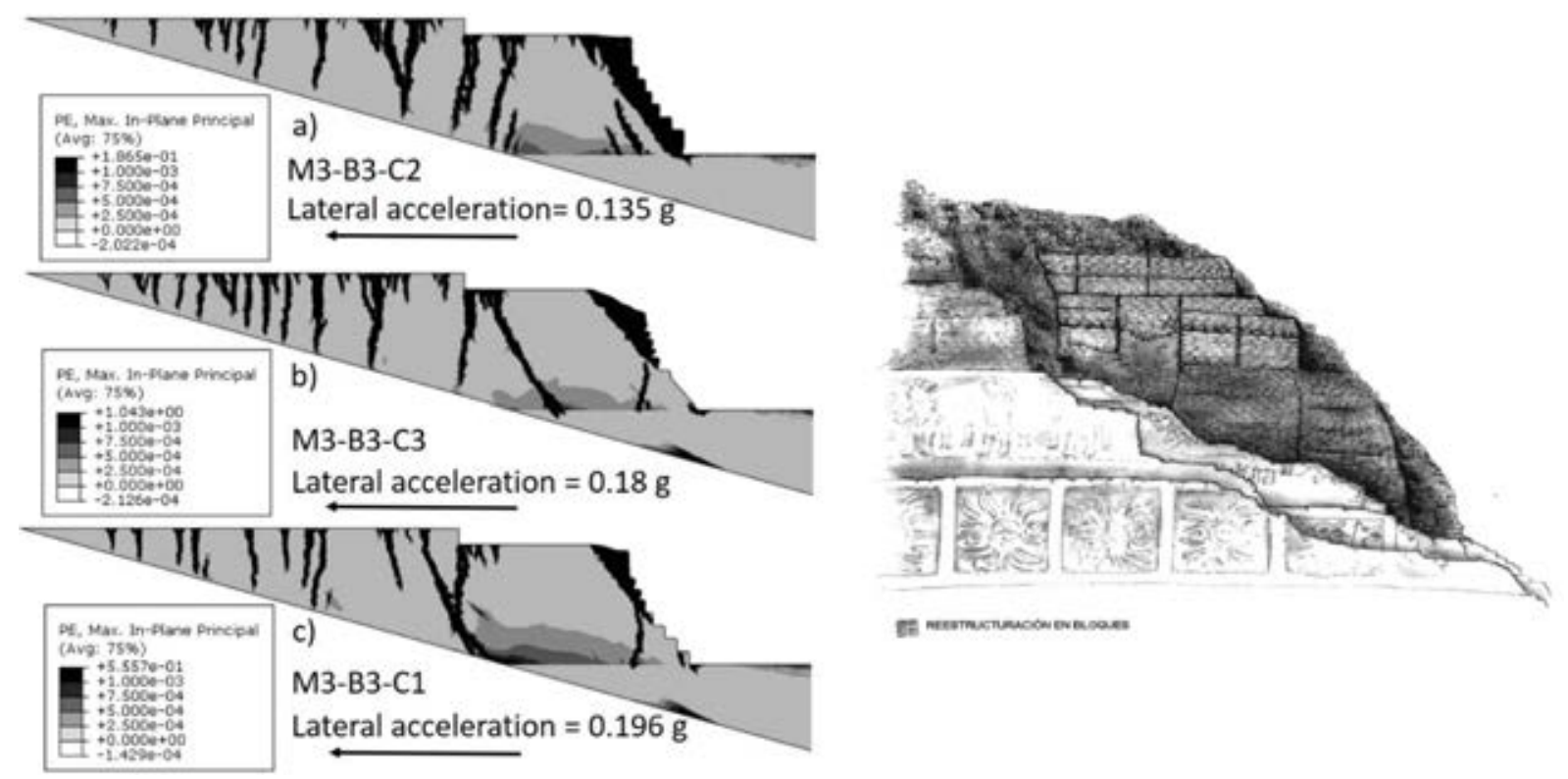

Figure 14: Lateral resistance of M3-B3 cases for step architecture variation (left), damage status of the NW corner of the main pyramid (right) [16].

\section{DISCUSSION}

Results indicate that under the assumptions made for material properties, bedrock and soil stratification, and architectural configuration, the 2D plane strain model shows localized material damage but does not collapse under gravitational loading. For each adobe tensile strength considered the bedrock configuration produces a major effect on the extension of the damaged areas, while the step architecture affects damage only marginally. Within the bedrock configurations, B2 is characterized by the largest structure-soil contact, thus inducing higher displacements and strain energy for the pyramid, but less damage then the B3 configuration. Although B3 yields less structure-soil contact, it assumes a steeper bedrock angle than B1 and B2, resulting in a deeper amount of soft soil under the west side of the pyramid.

The lateral acceleration tests indicate that the pyramid will reach failure conditions characterized by the extensive collapse of the west side under eastward accelerations ranging from $0.135 \mathrm{~g}$ to $0.24 \mathrm{~g}$. The lowest value is due to the combined effect of lowest adobe tensile strength (M3) with steeper bedrock angle (B3) and shorter step footprint on the west side (C2). Although for the M3-B3 set the step architecture appears to contribute marginally to structural stability under gravitation, it affects significantly its lateral capacity. The $\mathrm{C} 3$ and $\mathrm{C} 1$ configurations increase lateral capacity from $0.135 \mathrm{~g}$ under $\mathrm{C} 2$ to $0.18 \mathrm{~g}$ and $0.196 \mathrm{~g}$ for $\mathrm{C} 3$ and $\mathrm{C} 1$, respectively. As expected in the case of a massive earthen structure, the material tensile strength plays a major - if not dominant -role in determining the lateral capacity. Replacing the lowest tensile strength (M3) adobe in the B3-C2 configuration with the highest tensile strength (M1) increases the lateral capacity from $0.135 \mathrm{~g}$ to $0.24 \mathrm{~g}$ (a $78 \%$ increment).

In conclusion, the results of the present preliminary study can be tentatively extrapolated to the actual pyramid to suggest that the uneven structure/soft soil/ bedrock interaction due to the pyramid location is not likely to cause the west side structural collapse under static conditions. Under this perspective, the extensive structural damage present in the NW corner could be at- 
tributed to lateral accelerations produced by seismic actions rather than soft soil support conditions as initially suspected. However, given the inherent limitations of the $2 \mathrm{D}$ plane strain formulation, the characteristic features of Moche building techniques ignored in the present continuum model, and, most importantly, the uncertainties of the actual NS soil stratifications, these are still tentative considerations to be used as a point of departure for future in depth studies.

\section{REFERENCES}

[1] L.J. Castillo, S. Uceda, The Mochicas. Handbook of South American Archaeology, Springer, New York, 707-729, 2008.

[2] S. Uceda, R. Morales, Moche: Pasado y presente. Patronato del Valle de Moche, Trujillo, Peru, 2010.

[3] R. Aguilar, G. Zavala, B. Castañeda, S. Lopez, S. Retamozo, M. Montesinos, M.A. Pando, Y. Dong, R. Perucchio, Structural damage assessment of Huaca de la Luna, Perú: Preliminary results from ongoing multidisciplinary study. $10^{\text {th }}$ International conference on Structural Analysis of Historical Constructions: Anamnesis, diagnosis, therapy, controls (SAHC 2016), Leuven, Belgium, Sep. 13-15, 465-472, 2016.

[4] E. Ramírez, M. Montesinos, R. Marques, R. Morales, S. Uceda, P.B. Lourenço, R. Aguilar, Análisis mecánico de albañilería arqueológica de adobe bajo cargas de compresión uniaxial: El caso de Huaca de la Luna. $3^{\text {rd }}$ International Conference on Mechanical Models in Structural Engineering, Sevilla, Spain, June 24-26, 77-90, 2015.

[5] R. Aguilar, M. Montesinos, S. Uceda, Mechanical characterization of the structural components of Pre-Columbian earthen monuments: Analysis of bricks and mortar from Huaca de la Luna in Perú. Case Studies in Construction Material, 6, 16-28, 2017.

[6] G. Zavala, S. López, M. Pando, J. Zegarra, R. Morales, S. Uceda, B. Castañeda, R. Aguilar, Geotechnical and geophysical exploration in archaeological heritage: Initial assessment at Huaca de la Luna. $15^{\text {th }}$ Panamerican Conference on Soil Mechanics and Geotechnical Engineering, Buenos Aires, Argentina, 557-564, 2015.

[7] R. Aguilar, M. Pando, C. Briceno, G. Zavala, B. Casteñada, R. Perucchio, S. Uceda, Structural and geotechnical engineering assessment of Huaca de la Luna-A massive earthen Moche culture pyramid in Northern Peru. Journal of Cultural Heritage, 34, 8394, 2018.

[8] P. Roca, P. B. Lourenço, Introduction to masonry mechanics and modeling techniques. Advanced Master in Structural Analysis of Historical Constructions and Monuments Lecture Notes, Spain, 2008.

[9] P. B. Lourenço, Recent advances in masonry modelling: micro-modelling and homogenization. U. Galvanetto, M.H. Ferri Alibadi eds. Multiscale Modelling in Solid Mechanics: Computational Approaches, Imperial College Press, London, England, 2010.

[10] Abaqus Analysis Theory Manual Simulia v.6.14.4.5.2 Damage plasticity model for concrete and other quasi-brittle materials. Dassault System Corporation, Providence, RI, 2019. 
[11] Abaqus Analysis Theory Manual Simulia v.6.14.23.3.3 Inelastic behavior, Mohr-Coulomb. Dassault System Corporation, Providence, RI, 2019

[12] S. Tezcan, N. Tambe, C. Muir, R. Aguilar, R. Perucchio, Nonlinear FE analysis of the response to lateral accelerations of the triumphal arch of the church of Andahuaylillas, Peru. R. Aguilar et. al. eds. $11^{\text {th }}$ International Conference of Structural Analysis of Historical Constructions (SAHC 2018), Cusco, Peru, Sep. 11-13, 2018.

[13] J. Sun, S. Tezcan, R. Perucchio, The structural function of the Dutch buttressing of the east curtain wall of Elmina Castle, Elmina, Ghana. P. Roca, L. Pelà and C. Molins eds. $12^{\text {th }}$ International Conference on Structural Analysis of Historical Constructions (SAHC 2021), Barcelona, Spain, Sep. 29 - Oct. 1, 2021.

[14] Proyecto Arqueológico Huaca de Sol y de la Luna, Planta y Cortes Huaca de la Luna: Secciones y elevaciones cotas actuales. AutoCAD documents, 2013.

[15] S. Uceda, E. Mujica, R. Morales, Proyecto Arqueológico Huaca de Sol y de la Luna: Investigaciones en la Huaca de la Luna 2000. Facultad de Ciencias Sociales, Universidad Nacionales de Trujillo, 2006.

[16] S. Uceda, R. Morales, Proyecto Arqueológico Huaca de Sol y de la Luna: Informe Técnico 2002, Facultad de Ciencias Sociales, Universidad Nacionales de Trujillo, 2003. 